Reprod. Nutr. Develop., 1988, 28 (3 A), 573-588

\title{
Comportement alimentaire et état de réplétion du réticulo-rumen chez le mouton nourri à volonté de foin de prairie ou de luzerne, avec accès continu ou limité : incidences sur le contrôle physique de l'ingestion
}

\author{
R. BAUMONT, J. P. DULPHY, J. P. ANDRIEU \\ avec la collaboration technique de L. L'Hotelier, M. Jailler, M. Dudilieu
}

Station de Recherches sur la Nutrition des Herbivores, I.N.R.A., Theix, 63122 Ceyrat, France.

Summary. Feeding behavior and reticulo-ruminal fill in sheep fed ad libitum grass hay or lucerne hay with continuous or restricted access : effects on physical control of feed intake.

1) The effects of limiting time of access to forage (twice $1 \mathrm{hr} 30 \mathrm{~min} v s$ continuous access) on feeding behavior and reticulo-ruminal fill were studied with two hays : a late cut grass hay and an early second cut lucerne hay.

2) With continuous access to forage, voluntary intake of lucerne hay was higher $(1693 \mathrm{~g}$ dry matter (DM) per day) than that of grass hay (974 g DM per day). Lucerne hay was eaten more rapidly and needed less mastication per g DM. With limited time of access, eating rates were increased, but voluntary intake was maintained only with the grass hay. 3) With continuous access to forage, rumen pool sizes (RPS) of fresh matter, DM, organic matter $(O M)$, and fibre were higher after the evening main meal than after the morning main meal. RPS were always lower with lucerne hay, except after the evening meal. With limited time of access, RPS after the morning meal were higher than those reached by continuous access, but this was not true after evening meal.

4) The net removal of DM and $O M$, but not of fibre were increased during the main meal, and turnover rates of DM, OM and fibre were higher for lucerne hay.

5) These results confirm that the maximum degree of rumen fill is involved in the control of voluntary intake. However, rumen fill does not seem to control intake during the whole day and other factors may be involved, specially with rapidly digestible hays.

\section{Introduction.}

Il est communément admis, depuis la synthèse de Balch et Campling (1962), que la quantité de fourrage ingérée par le ruminant peut être limitée par l'état de réplétion du réticulo-rumen $(R R)$. Dans son modèle de régulation à court terme de l'ingestion, Forbes (1980) introduit une limitation physique liée à l'encom- 
brement du RR, la fin du repas pouvant être provoquée soit par satiété métabolique, soit lorsque cette limite est atteinte. Récemment, Dulphy et Faverdin (1987) ont discuté l'importance du rôle de l'état de réplétion du RR dans la régulation de l'ingestion chez le ruminant. En effet, certaines observations tendent à nuancer ce rôle, même avec des fourrages grossiers. Thomson et al. (1985) observent chez le mouton au pâturage une quantité de matière fraîche et de matière sèche dans le rumen plus élevée le soir que le matin, ce qui montre que le ruminant ne réagit pas de la même façon à l'état de réplétion du RR selon le moment de la journée. D'autre part, le niveau de contenu ruminal atteint à la fin d'un grand repas pourrait être augmenté en redonnant à l'animal un fourrage très appétible (Gatel, 1984). Par ailleurs, les signaux métaboliques responsables de la satiété à court terme sont encore relativement mal connus chez le ruminant et notamment, le rôle des AGV reste discutable. Duranton et Buéno (1985) ont montré chez le mouton que la production d'AGV liée à l'ingestion de $500 \mathrm{~g}$ de concentré ne modifie pas la consommation de foin à court terme $(3 \mathrm{~h})$, alors que la consommation journalière de foin est réduite. Si Anil et Forbes (1980), consécutivement à l'infusion d'AGV dans la veine porte, observent une réduction de l'ingestion, dose dépendante, chez le mouton, de Jong et al. (1981) n'ont pas obtenu d'effet chez la chèvre à des doses physiologiques.

Le rôle de l'état de réplétion du RR dans le contrôle de l'ingestion a souvent été étudié par accroissement artificiel de l'encombrement, soit par des vessies remplies d'eau (Campling et Balch, 1961 ; Grovum, 1979), soit par l'introduction de particules indigestibles (Welch, 1967 ; Welton et Baumgardt, 1970) ou encore par transfert de contenu ruminal provenant d'un animal donneur (Carr et Jacobson, 1967). Une autre méthode consiste à limiter le temps d'accès de l'animal au fourrage pour obliger l'animal à utiliser au maximum la capacité du RR lors de la période d'accès au fourrage.

Dans ce travail nous avons étudié, chez le mouton, les effets d'une réduction du temps d'accès au foin sur le comportement alimentaire et mérycique et sur l'évolution au cours de la journée de l'état de réplétion du RR, mesuré par la méthode des vidages manuels. Cette limitation du temps d'accès a été étudiée avec deux foins d'ingestibilités très différentes, un foin de prairie naturelle et un foin de luzerne, afin de se placer dans deux situations opposées.

\section{Matériel et méthodes.}

\section{Expérience 1.}

Six moutons castrés de race Texel âgés de 2-3 ans et d'un poids compris entre 55 et $60 \mathrm{~kg}$ ont reçu successivement un foin de prairie naturelle récolté en début de floraison, puis un deuxième cycle précoce de luzerne, selon le schéma expérimental suivant :

- Période 1 : foin de prairie avec accès continu entre les distributions ( $A C$ ) : - Période 2: foin de prairie avec retrait des refus $1 \mathrm{~h} 30$ après chaque distribution $(A L)$; 
- Période 3 : foin de luzerne avec accès continu entre les distributions (AC) ; - Période 4: foin de luzerne avec retrait des refus 1 h 30 après chaque distribution ( $A L)$.

Durant toute l'expérience les animaux ont été alimentés à volonté $(110 \%$ de la quantité ingérée la veille) avec deux distributions par jour à $8 \mathrm{~h}$ et à $16 \mathrm{~h}$, les refus étant enlevés chaque matin à $8 \mathrm{~h}$. L'éclairage a été maintenu constant à $16 \mathrm{~h}$ par jour (de $6 \mathrm{~h}$ à $22 \mathrm{~h}$ ) tout au long de l'expérience.

Les périodes 1 et 3 ont duré 3 semaines avec deux semaines d'adaptation au foin et une semaine pour la mesure des quantités ingérées, du comportement alimentaire (5 jours consécutifs) et de la digestibilité (par collecte totale des fèces durant 6 jours consécutifs). Durant les périodes 2 et 4 ces mesures ont été faites après une semaine d'adaptation au temps limité.

Mesure en continu de la quantité ingérée et des activités alimentaires et méryciques. - L'enregistrement en continu de la quantité ingérée a été obtenu par un système d'auges placées sur des capteurs métalliques intégrant toute variation de poids grâce à la présence de jauges de contrainte. Ces capteurs, munis d'amplificateurs, transmettent un signal électrique compris entre 0 et 5 volts. Un convertisseur analogique-digital associé à une interface de communication permet de recueillir les données sur un micro-ordinateur portable (EPSON HX 20) programmé de manière à pouvoir scruter en permanence les six auges placées sur les cages à digestibilité. Simultanément, les activités alimentaires et méryciques ont été enregistrées avec les appareils électroniques portables développés par Brun, Prache et Béchet (1984). L'utilisation d'un ballonnet rempli de mousse de polyéthurane maintenu par un licol en position sous-maxillaire permet d'améliorer la fiabilité des enregistrements. Le signal de pression ainsi obtenu est transformé en signal électrique par un microphone à capacité variable.

Des logiciels spécialement conçus à cet effet permettent d'interpréter les enregistrements des activités alimentaires et méryciques (Marzin, résultats non publiés) ainsi que de déterminer, à partir des enregistrements de la quantité ingérée, les durées et volumes des différents repas. Les repas et les périodes de rumination sont définis comme des séquences respectivement d'ingestion et de rumination sans interruptions supérieures à $5 \mathrm{~min}$. Les « grands repas » sont les repas consécutifs aux distributions du fourrage. Les " petits repas » sont les repas spontanés effectués entre les distributions et dont la durée a toujours été inférieure à celle des « grands repas".

\section{Expérience 2.}

L'évolution au cours de la journée de l'état de réplétion du RR a été suivie sur trois autres moutons, d'un poids compris entre 75 et $80 \mathrm{~kg}$, munis de fistules de $75 \mathrm{~mm}$ de diamètre et ayant suivi en tout point le même schéma expérimental.

Mesure de l'état de réplétion du $R R$. - Durant chaque période quatre vidages manuels complets du RR ont été réalisés sur chaque animal en les répartissant sur deux semaines afin de pouvoir observer un intervalle de $72 \mathrm{~h}$ entre deux vidages consécutifs sur le même animal de manière à ce qu'il n'y ait pas d'effet du premier 
vidage sur les valeurs mesurées lors du suivant (Aitchison, 1985). A chaque période les vidages ont été réalisés à : $8 \mathrm{~h}$ : avant la première distribution; $10 \mathrm{~h}$ : à la fin du grand repas du matin ; $16 \mathrm{~h}$ : avant la deuxième distribution ; $18 \mathrm{~h}$ : à la fin du grand repas du soir.

Afin de limiter l'incidence des variations journalières de quantité ingérée sur l'état de réplétion du $R R$, les animaux ont été limités la veille de chaque vidage à la quantité ingérée la veille du premier vidage plus $10 \%$. A chaque vidage un échantillon du contenu du réseau $(200 \mathrm{~g})$ a été prélevé afin d'en déterminer la teneur en matière sèche. Le poids frais du contenu total du RR a été pesé et, après homogénéisation, des échantillons de contenu de rumen ( 3 fois $250 \mathrm{~g}$ ) ont été prélevés afin de déterminer les teneurs en matière sèche (MS), matière organique (MO), ainsi qu'en NDF, ADF et ADL selon la méthode de Goering et Van Soest (1970). A chaque vidage la totalité du contenu (moins les échantillons prélevés) a été ensuite réintroduite dans le $R R$.

Estimation de la vitesse de disparition hors du RR et du taux de renouvellement du contenu du RR. - Pour un critère donné la vitesse de disparition horaire moyenne hors du RR entre deux points de cinétique consécutifs $\mathrm{Ta}$ et $\mathrm{Tb}$ est calculée ainsi :

$\left.V(\mathrm{Ta}, \mathrm{Tb})=\frac{1}{(\mathrm{~Tb}-\mathrm{Ta})} \times[\mathrm{Q}(\mathrm{Ta})+\mathrm{Q} \mathbf{( T a}, \mathrm{Tb})-\mathrm{Q}(\mathrm{Tb})\right]$

avec : $V(T a, T b)=$ vitesse de disparition entre $T a$ et $T b$

$\mathrm{Q}(\mathrm{Ta})$ et $\mathrm{Q}(\mathrm{Tb})=$ quantités présentes dans le $\mathrm{RR}$ à $\mathrm{Ta}$ et $\mathrm{Tb}$

Ql $(\mathrm{Ta}, \mathrm{Tb})=$ quantité ingérée entre $\mathrm{Ta}$ et $\mathrm{Tb}$.

A partir des vitesses de disparition, il est possible de calculer des taux de renouvellement moyens entre $\mathrm{Ta}$ et $\mathrm{Tb}$ :

$R(T a, T b)=2 \times \frac{V(T a, T b)}{Q(T a)+Q(T b)}$

et donc des taux de renouvellement moyens journaliers en faisant la moyenne pondérée.

\section{Analyses statistiques.}

Les effets mouton, nature du foin et temps d'accès ainsi que l'interaction entre les effets foin et temps d'accès ont été testés par analyse de variance. Dans les cas où l'interaction a été significative les comparaisons de moyennes ont été réalisées par le test de Student appliqué aux couples de données.

\section{Résultats.}

Composition chimique des deux foins utilisés. - La teneur en matières azotées du foin de luzerne (tabl. 1) était environ deux fois plus élevée que celle du foin de prairie, tandis que sa teneur en parois totales (NDF) était plus faible et que les teneurs en cellulose brute et ADF des deux foins n'étaient pas très différentes. La 
faible teneur en matières azotées totales ainsi qu'une teneur en parois végétales plus élevée du foin de prairie durant la période 2 indiquent une qualité légèrement moindre de ce foin durant la période à temps d'accès limité.

\section{TABLEAU 1}

Composition chimique des foins utilisés.

\begin{tabular}{|c|c|c|c|c|c|c|c|}
\hline & $\begin{array}{c}\text { Matière } \\
\text { sèche } \\
(\%)\end{array}$ & $\begin{array}{c}\text { Matière } \\
\text { organique }\end{array}$ & $\begin{array}{c}\text { Matières } \\
\text { azotées totales }\end{array}$ & $\begin{array}{l}\text { Cellulose } \\
\text { brute }\end{array}$ & $\begin{array}{l}\text { NDF } \\
\text { (en g/k }\end{array}$ & de mati & $\begin{array}{l}A D L \\
\text { sèche) }\end{array}$ \\
\hline $\begin{array}{l}\text { Foin de prairie } \\
\text { (AC) } \\
\text { (Période 1) }\end{array}$ & 87,6 & 926 & 84 & 307 & 635 & 362 & 44 \\
\hline $\begin{array}{l}\text { Foin de prairie } \\
\text { (AL) } \\
\text { (Période 2) }\end{array}$ & 89,4 & 932 & 64 & 328 & 671 & 384 & 50 \\
\hline $\begin{array}{l}\text { Foin de luzerne } \\
\text { (AC) } \\
\text { (Période } 3 \text { ) }\end{array}$ & 85,9 & 892 & 143 & 320 & 499 & 364 & 82 \\
\hline $\begin{array}{l}\text { Foin de luzerne } \\
\text { (AL) } \\
\text { (Période 4) }\end{array}$ & 84,9 & 893 & 152 & 324 & 503 & 372 & 81 \\
\hline
\end{tabular}

$A C=$ accès continu $; A L=$ accès limité $; N D F=$ neutral detergent fiber $; A D F=$ acid detergent fiber: $A D L=$ acid detergent lignin.

\section{Expérience 1.}

\section{Quantités ingérées totales et digestibilité.}

Les digestibilités de la MS et de la MO du foin de luzerne (tabl. 2) ont été supérieures de 4 à 5 points à celles du foin de prairie alors que la digestibilité des parois végétales a été voisine. La limitation du temps d'accès a entraîné une augmentation significative de 1 à 3 points des digestibilités de la MS et de la MO et de 4 à 5 points de la digestibilité des parois végétales pour les deux foins.

La quantité ingérée journalière moyenne (tabl. 3) de foin de luzerne (1 $693 \mathrm{~g}$ de MS en accès continu) a été nettement plus élevée que celle de foin de prairie ( $974 \mathrm{~g}$ de $\mathrm{MS}$ ). La limitation du temps d'accès à deux fois $1 \mathrm{~h} 30$ n'a pas eu d'effet pour le foin de prairie bien que la moindre qualité du foin de prairie durant la période 2 eût pu entraîner une diminution des quantités ingérées. Par contre, la consommation volontaire de foin de luzerne a été alors significativement réduite.

Les variations de quantités ingérées entre les animaux (écart-types de l'ordre de $200 \mathrm{~g}$ de MS pour les quatre périodes) expliquent que l'effet mouton soit significatif sur les mesures de digestibilité et sur la plupart des paramètres du comportement alimentaire (tabl. 3). 
TABLEAU 2

Influence de la nature du foin et de la limitation du temps d'access sur le poids vif des animaux et sur la digestibilité du fourrage (Expérience 1).

\begin{tabular}{lcccccc}
\hline & \multicolumn{2}{c}{ Foin de prairie } & \multicolumn{2}{c}{ Foin de luzerne } & $\begin{array}{l}\text { Ecart-type } \\
\text { résiduel }\end{array}$ & $\begin{array}{c}\text { Résultat de l'analyse } \\
\text { de variance }\end{array}$ \\
\cline { 2 - 4 } & $\mathrm{AC}$ & $\mathrm{AL}$ & $\mathrm{AC}$ & $\mathrm{AL}$ & & \\
\hline $\begin{array}{l}\text { Poids vif } \\
\text { des moutons }(\mathrm{kg})\end{array}$ & 56,3 & 55,8 & 59,3 & 59,5 & 1,21 & $\mathrm{~F}^{* *} ; \mathrm{M}^{* *}$ \\
$\begin{array}{l}\text { Digestibilité } \\
\text { (\% de l'ingéré) }\end{array}$ & & & & & & \\
$\begin{array}{l}\text { Matière sèche } \\
\text { Matière organique }\end{array}$ & 57,1 & 58,1 & 61,9 & 64,6 & 2,00 & $\mathrm{~F}^{* *} ; \mathrm{TA}^{*} ; \mathrm{M}^{*}$ \\
NDF & 51,6 & 59,2 & 62,1 & 65,4 & 2,01 & $\mathrm{~F}^{* *} ; \mathrm{TA}^{*} ; \mathrm{M}^{*}$ \\
ADF & 59,8 & 55,1 & 49,7 & 54,0 & 2,65 & $\mathrm{TA}^{* *} ; \mathrm{M}^{*}$ \\
& 49,8 & 54,6 & 2,70 & $\mathrm{TA}^{* *} ; \mathrm{M}^{*}$ \\
\hline
\end{tabular}

$A C=$ accès continu $; A L=$ accès limité $; N D F=$ neutral detergent fiber $; A D F=$ acid detergent fiber : $F=$ effet foin ; TA $=$ effet temps d'accès $: M=$ effet mouton : ${ }^{*}=(P<0,05) ;{ }^{* *}=(P<0,01)$.

\section{Prises alimentaires.}

En accès continu, le profil alimentaire des animaux se caractérise par un grand repas suivant la distribution, puis par une succession de petits repas et de périodes de rumination jusqu'à la distribution suivante. La différence d'ingestion journalière entre les deux foins a été due pour $95 \%$ à la différence de quantité ingérée lors des grands repas (tabl. 3). Avec le foin de luzerne la durée des grands repas a été allongée ( $+23 \mathrm{~min}$ le matin, $+37 \mathrm{~min}$ le soir), alors que la durée d'ingestion sous forme de petits repas est restée identique. Mais cette augmentation de la durée d'ingestion ( + $60 \mathrm{~min}$ ) n'explique pas à elle seule la différence des quantités ingérées. La vitesse d'ingestion a été également plus élevée avec le foin de luzerne. Cet accroissement de la vitesse d'ingestion a été plus important durant les grands repas $(+46 \%)$ que durant les petits repas $(+18 \%)$, l'ingestion durant ces derniers étant toujours nettement plus lente. Le pourcentage d'activité durant les repas (\% du temps passé à mastiquer) a été le même pour les deux foins aussi bien durant les grands que durant les petits repas. En revanche, durant les petits repas, ce pourcentage d'activité a été réduit de 7 points $(P<0,05)$ par rapport aux grands repas, ce qui indique une activité de recherche et de tri plus intense durant ceux-là.

Lorsque le temps d'accès a été limité à deux fois $1 \mathrm{~h} 30$, les animaux ont toujours utilisé la totalité du temps imparti à ingérer. Au bout de trois jours les animaux se sont adaptés au temps d'accès limité et la quantité ingérée journalière est demeurée stable. L'augmentation de la vitesse d'ingestion ( $+75 \%$ ) a permis, avec le foin de prairie, de maintenir la quantité ingérée journalière, alors qu'avec le foin de luzerne cette augmentation, pourtant du même ordre $(+71 \%)$, n'a pas suffi et la quantité ingérée journalière a été diminuée de $18 \%$. Le pourcentage 
TABLEAU 3

Influence de la nature du foin et de la limitation du temps d'accès sur les quantités ingérées et paramètres du comportement alimentaire (Expérience 1).

\begin{tabular}{|c|c|c|c|c|c|c|}
\hline & \multicolumn{2}{|c|}{ Foin de prairie } & \multicolumn{2}{|c|}{ Foin de luzerne } & \multirow{2}{*}{$\begin{array}{l}\text { Ecart-type } \\
\text { résiduel }\end{array}$} & \multirow{2}{*}{$\begin{array}{l}\text { Résultat de l'analyse } \\
\text { de variance }\end{array}$} \\
\hline & $A C$ & $\mathrm{AL}$ & $\mathrm{AC}$ & $A L$ & & \\
\hline \multicolumn{7}{|l|}{ Valeurs journalières } \\
\hline $\begin{array}{l}\text { Quantités ingérées } \\
\text { (g) }\end{array}$ & 974 & 1003 & $1693 \mathrm{~A}$ & $1380 \mathrm{Bb}$ & 58 & $\mathrm{~F}^{* *} ; \mathrm{TA}^{* *} ; \mathrm{M}^{* *} ; \mathrm{I}^{* *}$ \\
\hline $\begin{array}{l}\text { Durée d'ingestion } \\
\text { (min) }\end{array}$ & 304 & 173 & 364 & 172 & 27 & $F^{*} ; M^{*}$ \\
\hline $\begin{array}{l}\text { Vitesse d'ingestion } \\
(\mathrm{g} / \mathrm{min})\end{array}$ & 3,30 & 5,77 & 4,69 & 8,04 & 0,55 & $\mathrm{~F}^{* *} ; \mathrm{TA}^{* *} ; \mathrm{M}^{* *}$ \\
\hline $\begin{array}{l}\text { Durée de rumination } \\
\text { (min) }\end{array}$ & 580 & $642 a$ & 607 & 605 & 26 & $\mathrm{TA}^{*} ; \mathrm{M}^{* *} ; \mathrm{I}^{*}$ \\
\hline
\end{tabular}

\section{Grands repas}

Quantités ingérées

(g)

Durée active (min)

$\%$ d'activité

$772 \quad 1003$ a $1455 \mathrm{~A} \quad 1380 \mathrm{Bb}$

65

$F^{* *} ; \mathrm{TA}^{* *} ; \mathrm{M}^{* *} ; \mathrm{I}^{* *}$

Vitesse moyenne

$(\mathrm{g} / \mathrm{min})$

215

173

274

172

24

$F^{* *} ; M^{*}$

$92,2 \quad 94,1 \quad 92,9$

94,0

1,4

$\mathrm{TA}^{*} ; \mathrm{M}^{*}$

3,69

5,77

5,37

8,04

0,59

$F^{* *} ; \mathrm{TA}^{* *} ; \mathrm{M}^{* *}$

\section{Petits repas}

Quantités ingérées

(g)

Durée active ( $\mathrm{min}$ )

$\%$ d'activité

Nombre

Vitesse moyenne

$(\mathrm{g} / \mathrm{min})$

$\begin{array}{cccccl}202 & / & 238 & / & 101 & \text { N.S. } \\ 90 & / & 90 & / & 32 & \text { N.S. } \\ 85,0 & / & 85,2 & / & 4,7 & \text { N.S. } \\ 4,4 & / & 5,3 & / & 1,2 & \text { N.S. } \\ 2,26 & / & 2,67 & / & 0,25 & F^{*}\end{array}$

\section{Rumination}

\begin{tabular}{|c|c|c|c|c|c|c|}
\hline $\begin{array}{l}\text { Nombre de périodes } \\
\text { Nombre de cycles }\end{array}$ & $\begin{array}{l}13,5 \\
652\end{array}$ & $\begin{array}{l}14,5 \mathrm{a} \\
714 \mathrm{a}\end{array}$ & $\begin{array}{l}15,1 \mathrm{~A} \\
709\end{array}$ & $\begin{array}{c}14,1 \mathrm{~b} \\
681\end{array}$ & $\begin{array}{l}0,5 \\
48\end{array}$ & $\begin{array}{l}\mathrm{F}^{*} ; \mathrm{M}^{* *} ; \mathrm{I}^{*} \\
\mathrm{M}^{* *} ; \mathrm{I}^{*}\end{array}$ \\
\hline $\begin{array}{l}\text { Durée moyenne des } \\
\text { cycles }\end{array}$ & 47,7 & 45,4 & 44,7 & 45,1 & 1,3 & $\mathrm{~F}^{*} ; \mathrm{M}^{* *}$ \\
\hline $\begin{array}{l}\text { Durée de mastication } \\
\text { mérycique (min) }\end{array}$ & 502 & $532 a$ & 522 & 506 & 19 & $M^{* *} ; 1^{*}$ \\
\hline $\begin{array}{l}\text { Nombre unitaire de } \\
\text { cycles (/g MSI) }\end{array}$ & 0,68 & 0,75 & 0,43 & 0.51 & 0,06 & $\mathrm{~F}^{* *} ; T A^{* *} ; \mathrm{M}^{* *}$ \\
\hline $\begin{array}{l}\text { Durée unitaire de } \\
\text { mastication méryci- } \\
\text { que (min/g MSl) }\end{array}$ & 0,53 & 0,56 & 0,31 & 0,38 & 0,04 & $\mathrm{~F}^{* *} ; \mathrm{TA} A^{*} ; \mathrm{M}^{* *}$ \\
\hline \multicolumn{7}{|l|}{ Mastication } \\
\hline \multirow{2}{*}{$\begin{array}{l}\text { Durée totale (min) } \\
\text { Durée unitaire } \\
\text { (min/g MSI) }\end{array}$} & 806 & 706 a & $886 \mathrm{~A}$ & $678 b$ & 49 & $\mathrm{TA}^{* *} ; \mathrm{I}^{*}$ \\
\hline & 0,85 & $0,74 \mathrm{a}$ & $0,53 \mathrm{~A}$ & $0,51 \mathrm{~B}$ & 0,05 & $\mathrm{~F}^{* *} ; \mathrm{TA}^{* *} ; \mathrm{M}^{* *} ; \mathrm{I}^{*}$ \\
\hline
\end{tabular}

$A C=$ accès continu; $A L=$ accès limité; $M S I=$ matière sèche ingérée; $F=$ effet foin ; TA $=$ effet temps d'accès ; $M=$ effet mouton $; I=$ interaction foin $\times$ temps d'accès ; ${ }^{*}=(P<0,05)$; ${ }^{* *}=(P<0,01)$. Si interaction significative : $A=$ effet foin $(P<0,05)$ période 1 contre $3 ; B=$ effet foin $(P<0,05)$ période 2 contre $4 ; a=$ effet temps d'accès $(P<0,05)$ période 1 contre période 2 ; $b=$ effet temps d'accès $(P<0,05)$ période 3 contre 4 . 
d'activité durant le repas a été légèrement, mais significativement, augmenté avec les deux foins.

\section{Rumination.}

Lorsque l'accès au foin a été continu, la durée journalière de rumination ainsi que le nombre de cycles et la durée de mastication mérycique (nombre de cycles $x$ durée moyenne des cycles) n'ont pas été affectés de manière significative par la nature du foin (tabl. 3). Par contre les valeurs unitaires de ces trois variables (c'est-à-dire exprimées par gramme de MS ingérée) ont été nettement plus faibles avec le foin de luzerne. Avec ce dernier la rumination a été plus fragmentée (nombre de périodes significativement plus élevé) et la durée moyenne des cycles a été plus courte de $3 \mathrm{~s}(\mathrm{P}<0,05)$.

La limitation du temps d'accès a entraîné avec le foin de prairie, les quantités ingérées ayant été maintenues, un accroissement significatif de tous les paramètres caractérisant la rumination, à l'exception de la durée moyenne des cycles. Avec le foin de luzerne, les quantités ingérées ayant été réduites, les valeurs journalières des paramètres de la rumination sont restées stables, mais les valeurs unitaires ont été augmentées significativement.

\section{Mastication totale.}

En accès continu, le temps total passé à mastiquer (ingestion + rumination) a été de $806 \mathrm{~min}$ avec le foin de prairie et de $886 \mathrm{~min}$ avec le foin de luzerne, la différence ayant été due pour $75 \%$ à une durée d'ingestion accrue avec le foin de luzerne (tabl. 3). La durée unitaire de mastication a été nettement plus faible avec ce dernier, à la fois du fait d'une vitesse d'ingestion plus élevée et d'une durée unitaire de mastication mérycique plus faible.

La limitation du temps d'accès a eu pour effet de réduire la durée totale de mastication de $101 \mathrm{~min}$ pour le foin de prairie et de $208 \mathrm{~min}$ pour le foin de luzerne, la durée unitaire de mastication ayant été diminuée de $13 \%$ avec le foin de prairie alors qu'elle est restée stable avec le foin de luzerne.

\section{Expérience 2.}

La réalisation de vidages complets du RR n'a pas eu de répercussions sur les quantités ingérées par les 3 moutons munis de fistules utilisés dans l'expérience 2. Leur niveau d'ingestion, plus élevé que celui observé dans l'expérience 1, est à rapprocher de leur poids vif plus important. De même, leur vitesse d'ingestion lors des grands repas a été plus élevée $(+18 \%$ avec le foin de prairie et $+13 \%$ avec le foin de luzerne). Aucun grand repas n'a dépassé 120 min durant les périodes avec accès continu, ce qui a permis de réaliser les vidages deux heures après les 
distributions alors que le grand repas était terminé au moins depuis $5 \mathrm{~min}$. Du fait de cette vitesse d'ingestion plus élevée, la limitation du temps d'accès n'a pas eu d'effet significatif sur les quantités ingérées par ces animaux, même si avec le foin de luzerne elles ont diminué de $5 \%$.

\section{TABLEAU 4}

Influence de la nature du foin et de la limitation du temps d'accès sur les quantités ingérées et l'état de réplétion du réticulo-rumen (Expérience 2).

\begin{tabular}{|c|c|c|c|c|c|c|c|}
\hline & & \multicolumn{2}{|c|}{ Foin de prairie } & \multicolumn{2}{|c|}{ Foin de luzerne } & \multirow{2}{*}{$\begin{array}{l}\text { Ecart-type } \\
\text { résiduel }\end{array}$} & \multirow{2}{*}{$\begin{array}{l}\text { Résultat de l'analyse } \\
\text { de variance }\end{array}$} \\
\hline & & $A C$ & $\mathrm{AL}$ & $A C$ & $A L$ & & \\
\hline \multicolumn{8}{|l|}{ Quantités ingérées: } \\
\hline (g de $M S)$ & $\begin{array}{c}\text { totales } \\
8 \mathrm{~h}-10 \mathrm{~h} \\
10 \mathrm{~h}-16 \mathrm{~h} \\
16 \mathrm{~h}-18 \mathrm{~h} \\
18 \mathrm{~h}-8 \mathrm{~h}\end{array}$ & $\begin{array}{c}1227 \\
576 \\
61 \\
474 \\
117\end{array}$ & $\begin{array}{c}1190 \\
627 \\
/ \\
564 \\
/\end{array}$ & $\begin{array}{c}1766 \\
629 \\
216 \\
832 \\
88\end{array}$ & $\begin{array}{c}1679 \\
801 \\
1 \\
878 \\
/\end{array}$ & $\begin{array}{c}96 \\
53 \\
74 \\
99 \\
140\end{array}$ & $\begin{array}{l}\mathrm{F}^{* *} \\
\mathrm{~F}^{*} ; \mathrm{TA}^{*} \\
\mathrm{~F}^{* *}\end{array}$ \\
\hline $\begin{array}{l}\text { Vitesse d'ingestion } \\
\text { du grand repas ( } \mathrm{g}\end{array}$ & $\begin{array}{l}\text { lors } \\
/ \mathrm{min})\end{array}$ & 4,37 & 6,61 & 6.09 & 9,33 & 0,72 & $\mathrm{~F}^{* *} ; \mathrm{TA}^{* *}$ \\
\hline \multicolumn{8}{|c|}{$\begin{array}{l}\text { Quantités présentes dans } \\
\text { le réticulo-rumen }(g) \text { : }\end{array}$} \\
\hline $\begin{array}{l}\text { Matière } \\
\text { fraîche }\end{array}$ & $\begin{array}{r}8 \mathrm{~h} \\
10 \mathrm{~h} \\
16 \mathrm{~h} \\
18 \mathrm{~h}\end{array}$ & $\begin{array}{l}10283 \\
12420 \\
12324 \\
13555\end{array}$ & $\begin{array}{l}10446 \\
13268 \\
11608 \\
12468\end{array}$ & $\begin{array}{rr}8230 & \\
9820 & 1 \\
9273 & \\
11984 & 1\end{array}$ & $\begin{array}{r}8690 \\
11542 \\
9934 \\
12186\end{array}$ & $\begin{array}{l}756 \\
633 \\
667 \\
678\end{array}$ & $\begin{array}{l}\mathrm{F}^{* *} \\
\mathrm{~F}^{* *} ; \mathrm{TA}^{*} ; \mathrm{M}^{*} \\
\mathrm{~F}^{* *} \\
\mathrm{M}^{*}\end{array}$ \\
\hline $\begin{array}{l}\text { Matière } \\
\text { sèche }\end{array}$ & $\begin{array}{r}8 \mathrm{~h} \\
10 \mathrm{~h} \\
16 \mathrm{~h} \\
18 \mathrm{~h}\end{array}$ & $\begin{array}{ll}1 & 021 \\
1 & 417 \\
1 & 240 \\
1 & 626\end{array}$ & $\begin{array}{l}1010 \\
1600 \\
1168 \\
1440 \mathrm{a}\end{array}$ & $\begin{array}{rr} & 912 \\
1 & 238 \\
1 & 062 \\
1 & 627\end{array}$ & $\begin{array}{ll} & 968 \\
1 & 495 \\
1 & 188 \\
1 & 788 \text { B }\end{array}$ & $\begin{array}{r}59 \\
77 \\
71 \\
103\end{array}$ & $\begin{array}{l}M^{*} \\
F^{*} ; T A^{* *} ; M^{*} \\
M^{*} \\
F^{*} ; M^{* *} ; I^{*}\end{array}$ \\
\hline $\begin{array}{l}\text { Matière } \\
\text { organique }\end{array}$ & $\begin{array}{l}8 \mathrm{~h} \\
10 \mathrm{~h} \\
16 \mathrm{~h} \\
18 \mathrm{~h}\end{array}$ & $\begin{array}{ll} & 925 \\
1 & 286 \\
1 & 116 \\
1 & 471\end{array}$ & $\begin{array}{ll} & 907 \\
1 & 456 \\
1 & 057 \\
1 & 299 \text { a }\end{array}$ & $\begin{array}{r}816 \\
1098 \\
949 \\
1443\end{array}$ & $\begin{array}{ll} & 863 \\
1 & 331 \\
1 & 061 \\
1 & 598 \mathrm{~B}\end{array}$ & $\begin{array}{l}53 \\
72 \\
67 \\
96\end{array}$ & $\begin{array}{l}F^{*} ; M^{*} \\
F^{* *} ; T A^{* *} ; M^{*} \\
M^{*} \\
F^{*} ; M^{* *} ; I^{*}\end{array}$ \\
\hline $\begin{array}{l}\text { Neutral detergent } \\
\text { fiber }\end{array}$ & $\begin{array}{r}8 \mathrm{~h} \\
10 \mathrm{~h} \\
16 \mathrm{~h} \\
18 \mathrm{~h}\end{array}$ & $\begin{array}{r}765 \\
1081 \\
898 \\
1211\end{array}$ & $\begin{array}{r}741 \\
1196 \\
876 \\
1015 a\end{array}$ & $\begin{array}{r}664 \\
875 \\
762 \\
1100 \mathrm{~A}\end{array}$ & $\begin{aligned} 691 \\
1027 \\
843 \\
1230 \mathrm{~B}\end{aligned}$ & $\begin{array}{l}39 \\
51 \\
51 \\
69\end{array}$ & $\begin{array}{l}F^{*} ; M^{* *} \\
F^{* *} ; T^{* *} ; M^{*} \\
F^{*} ; M^{* *} \\
M^{* *} ; I^{* *}\end{array}$ \\
\hline $\begin{array}{l}\text { Acid detergent } \\
\text { fiber }\end{array}$ & $\begin{array}{r}8 \mathrm{~h} \\
10 \mathrm{~h} \\
16 \mathrm{~h} \\
18 \mathrm{~h}\end{array}$ & $\begin{array}{l}451 \\
631 \\
534 \\
683\end{array}$ & $\begin{array}{l}432 \\
691 \\
503 \\
575 a\end{array}$ & $\begin{array}{l}483 \\
619 \\
547 \\
774 \mathrm{~A}\end{array}$ & $\begin{array}{l}504 \\
742 \\
608 \\
866 \mathrm{~B}\end{array}$ & $\begin{array}{l}27 \\
38 \\
40 \\
60\end{array}$ & $\begin{array}{l}F^{*} ; M^{* *} \\
T^{* *} ; M^{* *} \\
F^{*} ; M^{*} \\
F^{* *} ; M^{*} ; I^{*}\end{array}$ \\
\hline $\begin{array}{l}\text { Acid detergent } \\
\text { lignin }\end{array}$ & $\begin{array}{l}8 \mathrm{~h} \\
10 \mathrm{~h} \\
16 \mathrm{~h} \\
18 \mathrm{~h}\end{array}$ & $\begin{array}{r}85 \\
114 \\
108 \\
121\end{array}$ & $\begin{array}{r}87 \\
117 \\
92 \\
103\end{array}$ & $\begin{array}{l}156 \\
182 \\
162 \mathrm{~A} \\
224\end{array}$ & $\begin{array}{l}159 \\
221 \\
185 \mathrm{~B} \\
245\end{array}$ & $\begin{array}{l}13 \\
14 \\
12 \\
19\end{array}$ & $\begin{array}{l}\mathrm{F}^{* *} \\
\mathrm{~F}^{* *} ; \mathrm{TA}^{*} \\
\mathrm{~F}^{* *} ; \mathrm{M}^{*} ; \mathrm{I}^{*} \\
\mathrm{~F}^{* *} ; \mathrm{M}^{*}\end{array}$ \\
\hline
\end{tabular}

$A C=$ accès continu $; A L=$ accès limité $; M S I=$ matière sèche ingérée $; F=$ effet foin ; TA $=$ effet temps d'accès; $M=$ effet mouton; $I=$ interaction foin $\times$ temps d'accès ; ${ }^{*}=(P<0,05)$; ${ }^{*}=(P<0,01)$. Si interaction significative : $A=$ effet foin $(P<0,05)$ période 1 contre $3 ; B=$ effet foin $(P<0,05)$ période 2 contre $4 ; a=$ effet temps d'accès $(P<0,05)$ période 1 contre période 2 . 
Un des trois moutons utilisés dans cette expérience a présenté une quantité de contenu dans le RR plus faible en moyenne de 10,9\% par rapport à celles des deux autres, ce qui explique un effet mouton significatif sur la plupart des paramètres mesurés (tabl. 4).

Etat de réplétion du RR au cours de la journée.

Les teneurs en MS du rumen et du réseau ont été significativement plus élevées $(P<0,01)$ tout au long de la journée avec le foin de luzerne (fig. 1). Durant les grands repas la teneur en MS du rumen a augmenté fortement alors que celle du réseau a diminué. La limitation du temps d'accès n'a pas modifié significativement les teneurs en MS du rumen et du réseau. Les grands repas ont toujours entraîné une forte augmentation de l'état de réplétion du RR (fig. 2 et tabl. 4), mais l'état de réplétion maximum n'a été atteint, hormis durant la période 2 (foin de prairie en accès limité), qu'à $18 \mathrm{~h}$, soit après le repas du soir.
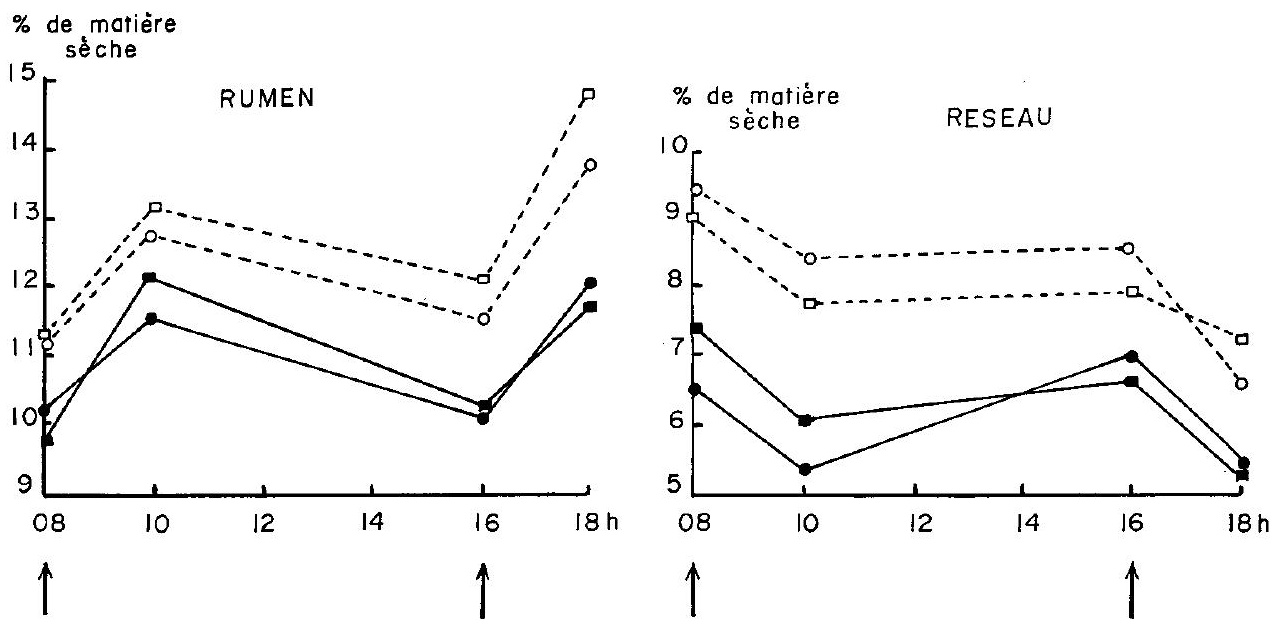

FIG. 1. - Evolution au cours de la journée de la teneur en matière sèche du rumen et du réseau; les animaux recevant successivement un foin de prairie en accès continu $(-)$, puis en accès limité ( - ensuite un foin de luzerne en accès continu (o-_o), puis en accès limité $(---)$.

La flèche $(\uparrow)$ indique la distribution du foin $(n=3)$.

Lorsque les animaux ont été alimentés en accès continu, la quantité de matière fraîche présente dans le $R R$ a été plus faible avec le foin de luzerne à tout moment de la journée, la différence ayant varié entre 1571 et $3051 \mathrm{~g}$ et étant significative à $8 \mathrm{~h}, 10 \mathrm{~h}$ et $16 \mathrm{~h}$. Malgré une teneur en MS du contenu de rumen 
plus élevée, les quantités de MS, de MO et de NDF ont été également plus faibles avec le foin de luzerne, même si le seuil de signification n'a pas été atteint à tout moment de la journée. A l'inverse les quantités présentes d'ADF dans le RR ont été légèrement supérieures avec le foin de luzerne et celles de résidu lignine (ADL) l'ont été nettement.

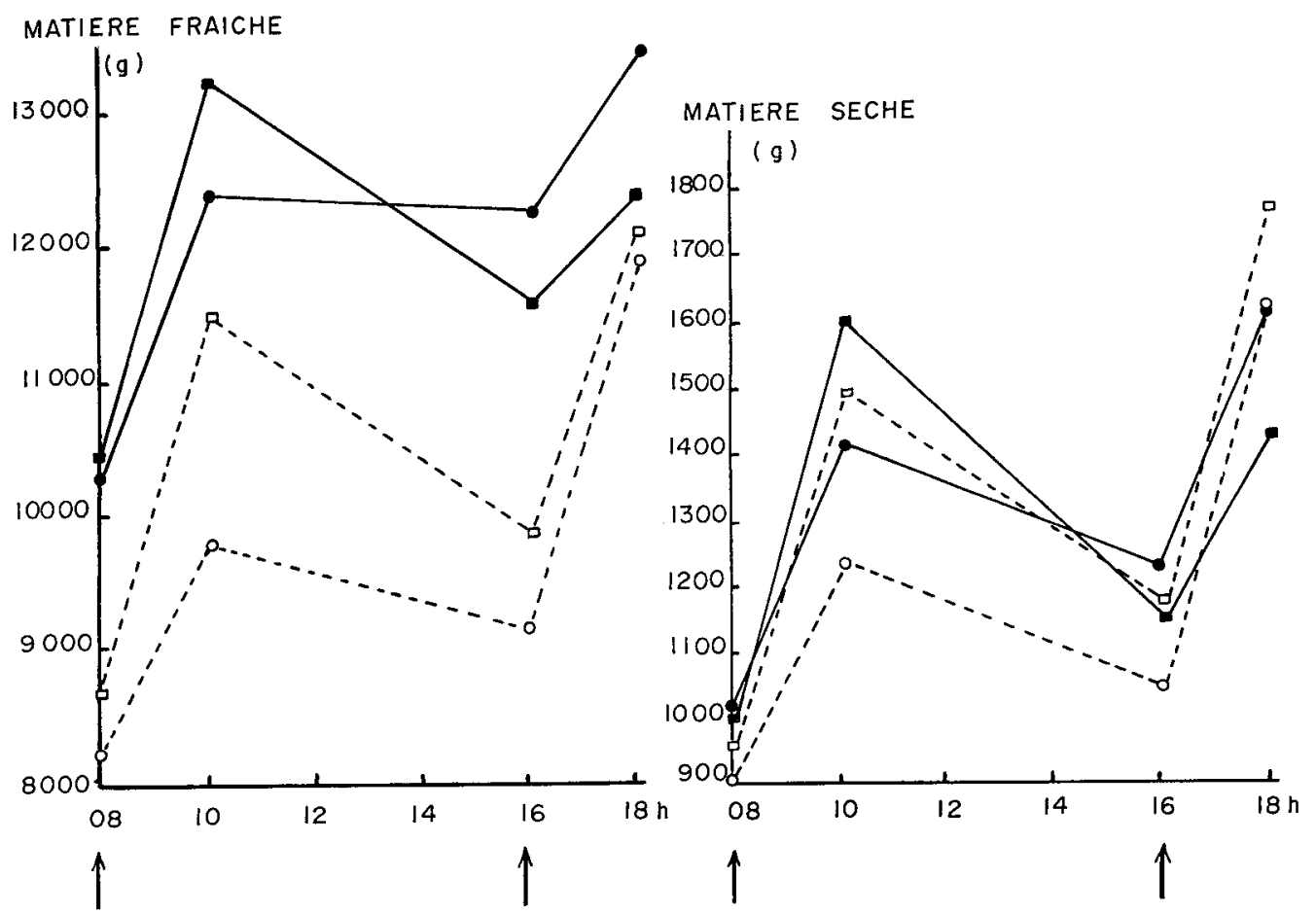

FIG. 2. - Evolution au cours de la journée des quantités de matière fraîche et de matière sèche présentes dans le réticulo-rumen; les animaux recevant successivement un foin de prairie en accès continu (-), puis en accès limité ( $\left.-\square_{-}\right)$et ensuite un foin de luzerne en accès continu $(\mathrm{o}-\mathrm{-}-\mathrm{O})$ puis en accès limité (-)

La flèche $(\uparrow)$ indique la distribution du foin $(n=3)$.

Les effets de la limitation du temps d'accès se sont manifestés surtout à $10 \mathrm{~h}$, soit après le grand repas du matin. L'état de réplétion du RR (fig. 2a) est alors proche de celui atteint en accès continu après le repas du soir. Toutes les quantités présentes dans le RR ont été alors significativement augmentées aussi bien avec le foin de prairie qu'avec le foin de luzerne par rapport à celles mesurées à $16 \mathrm{~h}$. De même, après le repas du soir, la quantité de matière fraîche présente dans le RR n'a pas été significativement différente de celle atteinte en accès continu, et les quantités de MS, MO ainsi que de parois végétales ont même été inférieures avec le foin de prairie. 
Vitesse de disparition hors du RR et taux de renouvellement.

A l'exception du repas du matin de la période 2 (foin de prairie en accès limité) les vitesses de disparition hors du RR de la MS et de la MO ont été fortement accélérées lors des grands repas (tabl. 5). Cet effet, lié à la prise de nourriture, a été plus important avec le foin de luzerne qu'avec le foin de prairie. En revanche, l'effet du grand repas a été très faible sur les vitesses de disparition des parois végétales et du résidu lignine $(A D L)$ et nous n'avons fait figurer que les valeurs moyennes dans le tableau 5 .

\section{TABLEAU 5}

Influence de la nature du foin et de la limitation du temps d'accès sur les vitesses de disparition et les taux de renouvellement des différents constituants du réticulo-rumen (Expérience 2).

\begin{tabular}{|c|c|c|c|c|c|c|c|}
\hline & & \multicolumn{2}{|c|}{ Foin de prairie } & \multicolumn{2}{|c|}{ Foin de luzerne } & \multirow{2}{*}{$\begin{array}{l}\text { Ecart-type } \\
\text { résiduel }\end{array}$} & \multirow{2}{*}{$\begin{array}{l}\text { Résultat de l'analyse } \\
\text { de variance }\end{array}$} \\
\hline & & $A C$ & $A L$ & $A C$ & $A L$ & & \\
\hline \multicolumn{8}{|c|}{$\begin{array}{l}\text { Vitesse de disparition : } \\
\text { (en } \mathrm{g} / \mathrm{h})\end{array}$} \\
\hline \multirow[t]{2}{*}{$\begin{array}{l}\text { Matière } \\
\text { sèche }\end{array}$} & $\begin{array}{r}8 \mathrm{~h}-10 \mathrm{~h} \\
10 \mathrm{~h}-16 \mathrm{~h} \\
16 \mathrm{~h}-18 \mathrm{~h} \\
18 \mathrm{~h}-8 \mathrm{~h}\end{array}$ & $\begin{array}{l}90 \\
39 \\
68 \\
52\end{array}$ & $\begin{array}{c}17 a \\
72 \\
146 a \\
31 a\end{array}$ & $\begin{array}{c}152 \mathrm{~A} \\
66 \\
134 \\
57\end{array}$ & $\begin{array}{c}137 \mathrm{~B} \\
51 \\
139 \\
59 \mathrm{~B}\end{array}$ & $\begin{array}{c}18 \\
10 \\
23 \\
4\end{array}$ & $\begin{array}{l}\mathrm{F}^{* *} ; \mathrm{TA}^{* *} ; \mathrm{I}^{*} \\
\mathrm{I}^{* *} \\
\mathrm{TA}^{*} ; \mathrm{I}^{*} \\
\mathrm{~F}^{* *} ; \mathrm{TA}^{* *} ; \mathrm{I}^{* *}\end{array}$ \\
\hline & moyenne & 53 & 50 & 74 & 70 & 3 & $\mathrm{~F}^{* *}$ \\
\hline $\begin{array}{l}\text { Matière } \\
\text { organique }\end{array}$ & $\begin{array}{l}\text { vitesse } \\
\text { moyenne }\end{array}$ & 49 & 46 & 65 & 62 & 3 & $\mathrm{~F}^{* *}$ \\
\hline NDF & $\begin{array}{l}\text { vitesse } \\
\text { moyenne }\end{array}$ & 34 & 35 & 37 & 35 & 2 & \\
\hline ADF & $\begin{array}{l}\text { vitesse } \\
\text { moyenne }\end{array}$ & 19 & 20 & 27 & 26 & 0,4 & $\mathrm{~F}^{* *}$ \\
\hline ADL & $\begin{array}{l}\text { vitesse } \\
\text { moyenne }\end{array}$ & 2,7 & 2,6 & 6,2 & 5,7 & 0,1 & $F^{* *}$ \\
\hline \multicolumn{8}{|c|}{$\begin{array}{l}\text { Taux de renouvellement } \\
(\text { en } \% / h)\end{array}$} \\
\hline \multicolumn{2}{|c|}{ Matière sèche } & 4,03 & 3,82 & $6,11 \mathrm{~A}$ & $5,14 \mathrm{Bb}$ & 0,16 & $\mathrm{~F}^{* *} ; \mathrm{TA}^{* *} ; \mathrm{M}^{* *} ; \mathrm{I}^{* *}$ \\
\hline \multicolumn{2}{|c|}{ Matière organique } & 4,17 & 3,94 & $6,12 \mathrm{~A}$ & $5,15 \mathrm{Bb}$ & 0,15 & $\mathrm{~F}^{* *} ; \mathrm{TA}^{* *} ; \mathrm{M}^{* *} ; \mathrm{I}^{* *}$ \\
\hline \multicolumn{2}{|c|}{ NDF } & 3,43 & 3,65 & $4,35 \mathrm{~A}$ & $3,73 \mathrm{~b}$ & 0,17 & $\mathrm{~F}^{* *}: \mathrm{M}^{* *} ; \mathrm{I}^{*}$ \\
\hline \multicolumn{2}{|l|}{ ADF } & 3,40 & 3,65 & $4,48 \mathrm{~A}$ & $3,82 \mathrm{~b}$ & 0,18 & $\mathrm{~F}^{* *} ; \mathrm{M}^{* *} ; \mathrm{I}^{* *}$ \\
\hline \multicolumn{2}{|l|}{ ADL } & 2,55 & 2,60 & $3,46 \mathrm{~A}$ & 2,84 & 0,15 & $\mathrm{~F}^{* *} ; \mathrm{TA}^{*} ; \mathrm{M}^{*} ; \mathrm{I}^{* *}$ \\
\hline
\end{tabular}

$A C=$ accès continu $; A L=$ accès limité $; N D F=$ neutral detergent fiber $; A D F=$ acid detergent fiber $; A D L=$ acid detergent lignin $; F=$ effet foin ; TA = effet temps d'accès; $M=$ effet mouton ; $I=$ interaction foin $\times$ temps d'accès; ${ }^{*}=(P<0,05) ;{ }^{* *}=(P<0,01)$.

$\mathrm{Si}$ interaction significative: $\mathrm{A}=$ effet foin $(\mathrm{P}<0,05)$ période 1 contre $3 ; \mathrm{B}=$ effet foin $(P<0,05)$ période 2 contre $4 ; a=$ effet temps d'accès $(P<0,05)$ période 1 contre période 2 ; $\mathrm{b}=$ effet temps d'accès $(P<0,05)$ période 3 contre 4 . 
Les vitesses horaires moyennes de disparition de la MS, de la MO, de I'ADF et de l'ADL, ainsi que leurs taux de renouvellement ont été significativement plus élevés avec le foin de luzerne. Si la vitesse de disparition des parois totales (NDF) a été comparable pour les deux foins, leur taux de renouvellement a été également plus élevé avec la luzerne puisque les quantités présentes de NDF dans le RR ont été alors plus faibles.

La limitation du temps d'accès a entraîné une diminution des vitesses de disparition de la MS lors du repas du matin, mais aucune des vitesses horaires moyennes de disparition hors du RR n'ont été modifiées significativement. Lorsque le temps d'accès a été limité, les taux de renouvellement n'ont pas été modifiés significativement avec le foin de prairie, même s'il y a eu une tendance à la baisse pour la matière sèche et la matière organique (moins $5 \%$ ). En revanche, ils ont été significativement réduits avec le foin de luzerne pour la MS, la MO, le NDF et l'ADF.

\section{Discussion.}

Dans les deux expériences, le foin de luzerne est ingéré en plus grande quantité que le foin de prairie et il est ingéré à la fois plus vite et plus longtemps durant les grands repas. L'étude des cinétiques d'ingestion durant le grand repas (Baumont, 1988) a montré que la vitesse initiale d'ingestion est également plus élevée pour le foin de luzerne, ce qui indique une meilleure appétibilité et Grenet (1986) a montré que la résistance à la mastication durant l'ingestion du foin de luzerne est moindre.

L'augmentation de la vitesse d'ingestion consécutive à la limitation du temps d'accès est conforme aux données obtenues sur vaches (Freer, Campling et Balch, 1962 ; Suzuki, Shinde et Hidari, 1970 ; Faverdin, 1985) et sur moutons (Hidari, 1981). Dans l'expérience 1, c'est avec le foin de prairie que les animaux ont été capables de maintenir la quantité ingérée journalière bien que la qualité du foin ait été légèrement moindre lorsque le temps d'accès a été limité. Avec le foin de luzerne en accès limité, les animaux ont vraisemblablement atteint leur vitesse d'ingestion maximale, ce qui peut expliquer la réduction de la quantité ingérée. En effet, dans l'expérience 2, les animaux ayant naturellement une vitesse d'ingestion plus élevée, la limitation du temps d'accès n'a pas affecté la consommation de foin de luzerne.

La limitation du temps d'accès entraîne une augmentation des activités méryciques, également observée chez la vache (Freer, Campling et Balch, 1962). Cette compensation peut s'expliquer par une mastication moins efficace lors de l'ingestion accélérée. Néanmoins, la durée unitaire de mastication totale est réduite en accès limité lorsque la quantité ingérée est maintenue (foin de prairie). En revanche, lorsque la limitation du temps d'accès entraîne une réduction de la quantité ingérée (foin de luzerne), la durée unitaire de mastication n'est pas modifiée, ce qui rejoint les données de Andrieu, Demarquilly et Korea (1987). 
Lorsque l'accès au foin est continu, les deux distributions étant espacées de $8 \mathrm{~h}$, l'état de réplétion maximum, que ce soit en matière fraîche ou en MS, n'est atteint que le soir, ce qui confirme les données obtenues par Dulphy, Béchet et Thomson (1975) avec des ensilages et par Thomson et al. (1985) au pâturage. L'apparition de la satiété lors du grand repas du matin intervient donc alors que l'encombrement du RR n'est pas maximum. En revanche, lorsque le temps d'accès est limité, l'état de réplétion après le repas du matin est plus proche du maximum atteint après le repas du soir en accès continu, mais ce maximum n'est pas dépassé en accès limité après le repas du soir. En accord avec Hidari (1981), il semble donc qu'il existe pour un aliment donné, un état de réplétion maximum, qui est atteint à la fin des périodes principales d'ingestion, soit après le repas du soir lorsque l'accès est continu, soit à la fin de la période d'accès à la ration lorsque celui-ci est limité dans le temps et que la vitesse d'ingestion n'est pas limitante. II serait intéressant, dans une expérience complémentaire, de ne réduire le temps d'accès que de la durée consacrée aux petits repas en accès continu.

$\mathrm{Si}$, pour des quantités ingérées égales, l'état de réplétion du $R R$ est nettement plus faible pour les bovins recevant un foin de luzerne (INRA, 1978) ainsi que pour des moutons recevant un foin de trèfle par rapport à un foin de ray-grass (Aitchison et al., 1986), nous obtenons un résultat similaire avec des animaux alimentés ad libitum et donc ingérant le foin de luzerne en plus grande quantité. L'encombrement du rumen ne semble donc pas être le seul facteur intervenant dans l'apparition de la satiété avec un foin de luzerne.

L'accélération de la vitesse de disparition hors du RR, de la MS et de la MO durant les grands repas est en accord avec les données de Reid et al. (1979), Moseley et Jones (1984), Aitchison et al. (1986), Grenet (1987). Cet effet de la prise de nourriture est certainement dû, d'une part à l'activation des fermentations durant le repas (Focant, 1984), ce qui semble confirmé par le fait que ces accélérations des vitesses de disparition soient plus importantes avec le foin de luzerne plus rapidement fermentescible, et d'autre part à l'augmentation du débit réticulo-omasal liée à la prise alimentaire (Buéno, 1975). La diminution importante de la teneur en MS du réseau durant les grands repas indique que le flux sortant du RR voit sa teneur en MS abaissée durant la prise de nourriture, ce qui confirmerait les données de Alderson et al. (1972), et pourrait être expliqué par un apport accru de salive et d'eau de boisson lors du grand repas.

La méthode utilisée pour le calcul des taux de renouvellement ne peut constituer qu'une approximation dans nos conditions expérimentales, puisqu'elle repose sur I'hypothèse d'une évolution linéaire de la quantité présente d'un constituant donné dans le RR entre deux points de cinétique. Néanmoins les valeurs calculées ici rejoignent celles obtenues par l'utilisation des marqueurs (Faichney, 1980 ; Poncet et al., 1986). Les taux de renouvellement plus élevés indiquent que la digestion dans le RR et le transit hors du RR sont nettement plus rapides avec le foin de luzerne, ce qui explique que l'état de réplétion du $R R$ soit moins important avec ce foin. La limitation du temps d'accès n'a pas eu d'effet sur les taux de renouvellement avec le foin de prairie, hormis une légère réduction pour la matière sèche et la matière organique qui peut s'expliquer par la baisse de qualité du foin. Par contre, la réduction des taux de renouvellement en accès limité 
avec le foin de luzerne montre bien que, pour un aliment donné, le taux de renouvellement dépend de la quantité ingérée, puisque cette dernière était alors plus faible.

L'augmentation de la digestibilité du foin de luzerne en accès limité semble être une conséquence de la réduction de la quantité ingérée puisque le temps de séjour dans le RR est alors plus long et que la durée unitaire de mastication reste stable. En revanche, l'augmentation de la digestibilité du foin de prairie en accès limité, et notamment des parois végétales, pourrait s'expliquer par une efficacité accrue du fonctionnement du RR, puisque les temps de séjour ne sont pas modifiés significativement et que la durée unitaire de mastication est alors réduite.

En conclusion, la limitation du temps d'accès à deux fois $1 \mathrm{~h} 30$ nous a permis de montrer que l'état de réplétion du RR, apprécié par la quantité présente de contenu, n'est pas en permanence au cours du nycthémère le signal de satiété prépondérant. D'autres facteurs doivent certainement intervenir dans l'établissement de la satiété, notamment lors du repas du matin ainsi qu'avec les fourrages rapidement digestibles comme le foin de luzerne. En accord avec Forbes (1986), il est donc nécessaire de considérer le contrôle de la prise alimentaire chez le ruminant comme un processus multifactoriel.

Reçu en juin 1987.

Accepté en décembre 1987

\section{Références}

AITCHISON E. M., 1985. A study of the removal of fibre from the rumen and voluntary intake of sheep eating hay diets. Ph. D. Thesis, Univ. of Reading.

AITCHISON E. M., GILL M., DHANOA M. S., OSBOURN D. F., 1986. The effect of digestibility and forage species on the removal of digesta from the rumen and the voluntary intake of hay by sheep. Brit. J. Nutr., 56, 463-476.

ALDERSON N. E., MITCHELL G. E., LITTLE J. C. O., CALL J. L., 1972. Post-prandial patterns of ovine rumino-reticular motility and digesta passage. J. anim. Sci., 35, 102-108.

ANDRIEU J., DEMARQUILLY C., KOREA A., 1987. Influence du niveau d alimentation sur les activités alimentaires et méryciques du mouton recevant des fourrages verts à différents stades de végétation. Reprod. Nutr. Dévelop., 27, 189-190.

ANIL M. H., FORBES J. M., 1980. Feeding in sheep during intraportal infusions of short-chain fatty acids and the effects of liver denervation. J. Physiol. Lond., 298, 407-414.

BALCH C. C., CAMPLING R. C., 1962. Regulation of voluntary food intake in ruminants. Nutr. Abstr. Rev., 32, 669-686.

BAUMONT R., 1988. Cinétique d'ingestion lors du grand repas chez le mouton : influence du temps d'accès au foin et de son ingestibilité. Reprod. Nutr. Dévelop., 28, 183-184.

BRUN J. P., PRACHE S., BECHET G., 1984. A portable device for eating behaviour studies. 5th Meet. Eur. Grazing Workshop.

BUÉNO L., 1975. Les fonctions motrices et digestives du feuillet. Thèse de Doctorat Es-Sciences. Université de Toulouse, $206 \mathrm{p}$.

CAMPLING R. C., BALCH C. C., 1961. Factors affecting the voluntary intake of food by cows. 1 - Preliminary observations on the effect, on the voluntary intake of hay, of changes in the amount of the reticulo-rumen contents. Brit. J. Nutr., 15, 523-530.

CARR S. B., JACOBSON D. K., 1967. Intraruminal addition of mass of removal of rumen contents on voluntary intake of the bovine. J. Dairy Sci, 50, 1814-1818. 
DE JONG A., STEFFENS A. B., DE RUITER L., 1981. Effects of portal volatile fatty acids infusions on meal patterns and bload composition in goats. Physiol. Behav., 27, 683-689.

DULPHY J. P., BÉCHET G., THOMSON E., 1975. Influence de la structure physique et de la qualité de conservation des ensilages de graminées sur leur ingestibilité. Ann. Zootech., 24, 81-94.

DULPHY J. P., FAVERDIN P., 1987. L'ingestion alimentaire chez les ruminants: modalités et phénomènes associès. Reprod. Nutr. Dévelop., 27, 129-155.

DURANTON A., BUENO L., 1985. Influence of regimen (roughage $v s$. concentrates) on satiety and forestomach motility in sheep. Physiol. Behav., 35, 105-108.

FAICHNEY G. J., 1980. Measurement in sheep of the quantity and composition of rumen digesta and of the fractional outflow rates of digesta constituents. Autr. J. agric. Res., 31, $1129-1137$.

FAVERDIN P., 1985. Régulation de l'ingestion des vaches laitières en début de lactation : variations au cours du nycthémère de l'activité alimentaire. des métabolites sanguins et de l'insulinémie : étude du rôle de l'insuline. Thèse de Doct. Ingén., INA-PG, 131 p.

FOCANT M., 1984. Comportement alimentaire, rumination, fermentations réticulo-ruminales et acides gras volatils plasmatiques, comparés chez la chèvre et le mouton ; influence du régime. Reprod. Nutr. Dévelop., 24, 239-250.

FORBES J. M., 1980. A model of the short-term control of feeding in the ruminant, effects of changing animal or feed characteristics. Appetite, 1, 21-24.

FORBES J. M., 1986. The voluntary food intake of farm animals. Butterworth \& Co Ltd., $206 \mathrm{p}$.

FREER M., CAMPLING R. C., BALCH C. C., 1962. Factors affecting the voluntary intake of food by cows. 4 - The behaviour and reticular motility of cows receiving diets of hay, oat straw and oat straw with urea. Brit. J. Nutr., 16, 279-295.

GATEL F., 1984. Signification de la satiété à court terme chez le mouton : influence de la qualité du fourrage et des stimuli associées à la prise de nourriture. Ann. Zootech., 33, 111-118.

GOERING H. K., VAN SOEST P. J., 1970. Forage fiber analyses. Agricultural Handbook no. 379. Washington, DC : US Department of Agriculture.

GRENET E., 1986. Comportement alimentaire comparé du mouton recevant des foins de graminées ou de légumineuses. Reprod. Nutr. Dévelop., 26, 273-274.

GRENET E., 1987. Etude comparée de la vitesse de vidange du rumen chez le mouton alimenté avec des foins de graminées et de légumineuses. Reprod. Nutr. Dévelop., 27, 229-230.

GROVUM W. L., 1979. Factors affecting the voluntary intake of food by sheep. 2 - The role of the tension and tactil input from compartments of the stomach. Brit. J. Nutr., 42, 425-436

HIDARI H., 1981. The relationships between rumen load and diurnal eating pattern of sheep fed in various time of access to feed. Jap. J. zootech. Sci, 52, 219-226.

INRA, 1978. Alimentation des ruminants. Ed. INRA Publications (Route de Saint-Cyr), 78000 Versailles, France, $597 \mathrm{p}$.

MOSELEY G., JONES J. R., 1984. The physical digestion of perennial rye-grass (Lolium perenne) and white clover (Trifolium repens) in the foregut of sheep. Brit. J. Nutr., 52, 381-390.

PONCET C., BEAUFORT M. T., AL ABD A., 1986. Vitesse de passage des résidus alimentaires et des liquides dans le tube digestif du mouton selon le marqueur et le type de ration. Reprod. Nutr. Dévelop., 26, $321-322$.

REID C. S. W., JOHN A., ULYATT M. J., WAGHORN G. C., MILLIGAN L. P., 1979. Chewing and the physical breakdown of feed in sheep. Ann. Rech. Vét., 10, 205-207.

SUSUKI S., SHINDE Y., HIDARI H., 1970. Effects of a change in the daily time of access to hay on the rate of eating and feed intake of dairy cows. Jap. J. zootech. Sci., 41, 423-429.

THOMSON R. F., CRUICKSHANK C. J., POPPI D.P., SYKES A. R., 1985. Diurnal petterns of rumen fill in grazing sheep. Proc. N.Z. Soc. anim. Prod., 45, 117-120.

WELCH J. G., 1967. Appetite control in sheep by indigestible fibers. J. anim. Sci., 26, 849-854.

WELTON R. F., BAUMGARDT B. R., 1970. Relative influence of palability on the consumption by sheep of diets diluted with 30 and 50 percent sawdust. J. Dairy Sci., 53, 1771-1774. 